Rev. Chll. Pediatr. 68 (1); 48-52, 1997

\title{
Tonometría gástrica en paciente pediátrico
}

\author{
Alejandro Donoso F. ${ }^{1,2}$; Mariø Cerda $S^{2}$ \\ Cristián Clavería R. '; Cristián Valverde G. ${ }^{1}$
}

\begin{abstract}
Resumen
Se describen los fundomentos térícos e iridicaciones de la tonomelria, ilusirándola con uno experiencia iniçial en e país. La torometr'c gásirica fiene cado vez más acogido como mélodo relativamerte simple y no invosivo para evaluar la magnitud ce la hipoxia tisular de í mucosa enteral, un reirilorio de gron mportancio eri pacientes en estado critco. E procedimiento, que es aplicable en paciertes pediáricos; permile estimar lo disponibilidad y ulilizasión de oxigeno en a mucoso gástrica a partir del pH en el inlerior de éla aplicondo la ecuoción de Henderson rossebalch modificado a partir de la rensión de dióxido de carbono en el interior del esiómago -obtenida por equilisrio en solución salina contenido es uno sondo balćn de silicona- y de la concentración arterial de bicarbonato. Anbas medicionzs se rea izan en un aralizcdor corriente de goses.
\end{abstract}

(Palabras clave: anoxic, oxigeno, anhidrido carbórico, choque, insuficiencia circularario periférica. tonometria gástrica, cuidados intensivos pedićtricos.l

\section{Estimating tissue oxigenation by gastric tonometry}

Principles and indications of gastriz lonmetry o:e cescribec togeher with o local experience on its use in 39 pediatric patienis 'o protote knowlegce and enhance its d nicol sppicotion in critically ill infents and children. Gastric tonometry is an interesting rion-invosive method to eva ucie oxigen metobolism in the gastrointestinal mucosa in critically Il: pat ents with circulatory failure. Mucosal oxigenatior. is estimaled from gastric intramucoscl pit calculations througr: a macified Herderson Hosselbac-s formula slark ng wiln sirutarieous measuremenks ó intra uminal gastric carbon dicxide ersion and ateriol bload bicarbonale concentration. The firs is obtained by equilibratig gastric corbon dicxide rension into nomgl soine solution containec in on appropriate, silicone welled, intagostric boloon catheter. Arrerial blood is samoled in the usual way for gas content onolysis. Ecth determinctiors require o standard blood gas onolyser

[Key words: cnoxia, carbon dioxide, oxvgen, $\mathrm{pH}$, gaslac tonomelry, srickk, pediorric, intensıve care unit.]

El manejo hemadinámico del paciente pediátrico críticamente enfermo ha sido tradicionalthente orientado por la objetivación de cambios en variables sistémicas globales y de expresión generalmente tardía (por ejemplo frecuencia cardíacn, perfusión periférica, diuresis, presión arterial), exámenes de laboratorio (como lactacidemia o indicadores de aporte y consumo de

1. Unidad de Cuidados Intensivos Pediátricos. Hospital Dr. Sótero del Río.

2. Unidad de Cuidados Intensivos Pediátricos. Clínica Alemana de Santiago. oxigeno. Sólo en un número limitado de casos se puede disponer de informacion sobre determinadas variables hemodinámicas medidas mediante catéteres percutáneos guiados por el flujo sanguíneo hasta la arteria pulmonar (Swan Ganz), pues el método es invasivo, tiene indicaciones precisas y requiere una infraestructura compleja y cara que no todos los hospitales nacionales pueden afrontar ${ }^{1}$. De aquí la tendencia a favorecer procedimientos para evaluar la oxjgenación regional de los distintos territorios corporales. con métodos menos invasivos y más accesibles a nuestras posibilidades locales ${ }^{2}$. En- 
tre ellos la determinación de la acidez mucosa gástrica. midiendo el $\mathrm{pH}$ intramucoso $(\mathrm{pHi})$, como expresión de la utilización de oxígeno en el teritorio esplác-nico, mediante el procedimiento denominado tonometría gástrica (TG).

El propósito de esta comunicación es contribuir a la difusión del conocimiento de la tonometría gástrica como método efectivo y sencillo de evaluación hemodinámica en pacientes pediátricos sometidos a cuidados intensivos, revisando sus fundamentos, interpretación e indicaciones, en base a la experiencia adquirida en nuestra unidad y otras de publicación teciente.

\section{Fundamentos fisiopatológicos}

Las características anatómicas y funcionales de la mucosa gastrointestinal permiten comprender la especial sensibilidad de la mucosa intestinal ante la vasoconstricción esplácnica en diversas situaciones clínicas que se caracterizan por entrega inadecuada de oxígeno de tejidos. Las principates son el carácter terminal de la circulación de la sangre en el extremo distal de las vellosidades; la gran cercanía de la rama aferente arterial y la eferente venosa en la base de Ia vellosidad, que establece un flujo sanguíneo de contracorriente, permitiendo algún "cortocircuito de oxígeno" en esta estructura, fenómeno que se acentúa si el flujo sanguíneo es bajo, pues aumenta el tiempo de difusión de oxígeno desde el lado arterial hacia el lado venoso. Otras caracteristicas importantes son el alto grado de recambio celular ${ }^{3}$ y el papel de barrera física, mecánica y metabólica de la pared intestinal, funciones que se alteran con la isquemia.

Por estos motivos el territorio esplácnico suele ser el primero en ser afectado y el último en recuperarse cuando ocurren alteraciones cardiovasculares y circulatorias con hipoxia tisular (choque séptico o cardiogénico, hipovolemia grave), produciéndose deficiente perfusión enteral que afecta muy precozmente a la mucosa, favoreciendo el paso de bacterias toxinas hacia la circulación general, con la consecuente activación de una respuesta inflamatoria. Esta, a su vez, precipita eventos que contribuyen a perpetuar la alteración circulatoria injcial y, si no son interrumpidos a tiempo, pueden conducir hacia falla orgánica múltiple $e^{4.5}$. De lo anterior se desprende la vital importancia de detectar y corregir prontamente tal situación, en la fase "compensada" del choque, situación frecuente pero rara vez apreciada en la realidad clínica.

La tonometría para determinar la acidez de la mucosa gástrica ( $\mathrm{pHi}$ ) y evaluar la oxigenación, por to tanto la magnitud de la isquemia local, ha sido refrendada por resultados clínicos y experimentales que indican que es valiosa en el seguimiento de pacientes con rjesgo alteraciones de las funciones cardiovasculares y circulatorias, pues la disminucion del pHi es un buen predictor de morbimortalidad y de falla orgánica múltiple, especialmente en pacientes adultos graves hospitalizados en unidades de cuidados intensivos.

\section{Estimación del pHi}

Los instrumentos para determinar el $\mathrm{pHi}$ en la mucosa gástrica, empleados en nuestra experiencia (figura 1), son los tonómetros TRIP, Tonometrics Inc. USA, que pueden ser de dos tjpos (gasstrico o sigmoideo), optándose por uno u otro según la edad del paciente. El tonómetro gástrico es de mayor tamaño y permite la aspiración simultánea de contenido del estómago. Están constituidos por una sonda impermeable en cuyo extremo distal hay un balón de silicona muy permeable a los gases, que se introduce en el estómago por vía oral o nasal, comprobando su ubicación mediante radiografia simple de abdomen. El balón de silicona se Jlena con solucion salina $0,9 \%(2,5 \mathrm{ml})$, evitando la entrada de aire, pues ello alteraría la medición del $\mathrm{CO}_{2}$ en la solución, manteniéndolo por un período variable, del orden de 30 minutos en nuestra experiencia, para permitir el equilibrio entre el $\mathrm{CO}_{2}$ de la mucosa gástrica y el de la solución salina. Simultáneamente se obtiene una muestra de sangre arterial por punción. Una vez cumplido el período de equilibrio de la sonda gástrica se desecha el primer $\mathrm{ml}$ de la solución, por corresponder al líquido del espacio muerto, y el resto (contenido del balón de silicona) se envía al taboratorio para medir la tensión de $\mathrm{CO}_{2}$ en un analizador corriente de gases, en nuestro caso un modelo Ciba Corning 238, al mismo tiempo que se mide la concentración de bicarbonato en la muestra de sangre arterial. 


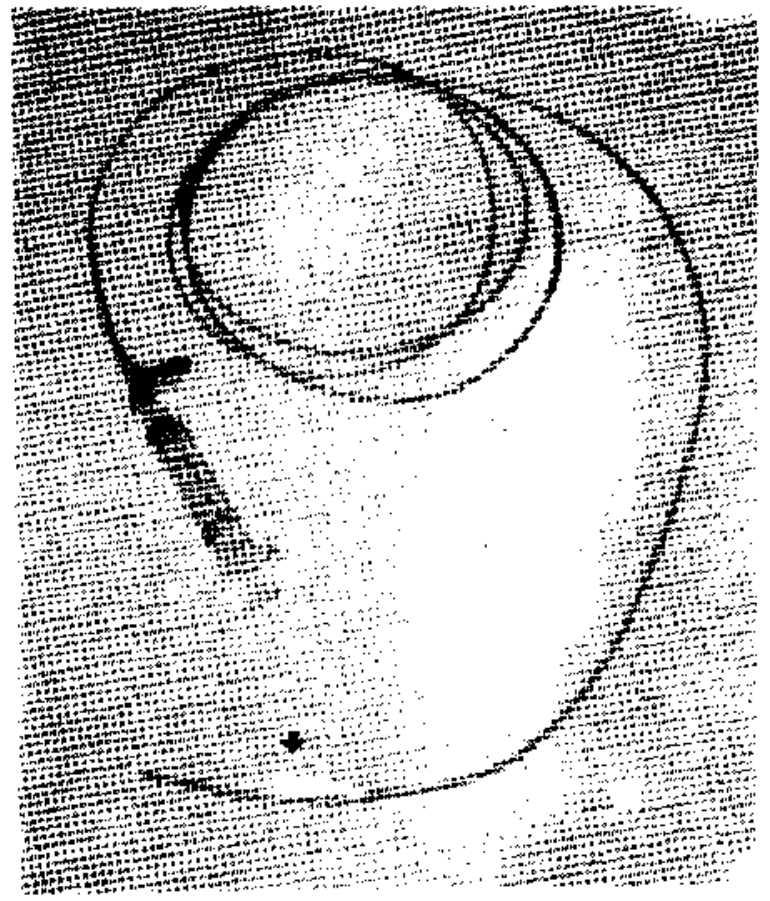

Figura 1: Tonúnetro sigmoideo con balón de silicona (tlechat lleno de solución fisiológica.
El pHi se estima indirectamente, asumiendo que la $\mathrm{pCO}_{2}$ del lumen intestinal es igual a la de la capa superficial de la mucosa gástrica, como también que la concentración de bicarbonato es semejante en el capilar sanguíneo gastrointes(inal y en la sangre arterial (figura 2$)^{7, B}$, por medio de la ecuación de Henderson Hasselbach modificada:

$\mathrm{pHi}=6,1+\log \left[\left(\mathrm{HCO}_{3}\right)\right.$ arterial $\left./ \mathrm{pCO}_{2}(\mathrm{ss}) \times 0.03\right]$

donde, $\mathrm{pCO}_{2}$ (ss) es la tensión de $\mathrm{CO}_{2}$ gásttica observada, multiplicada por un factor de correción según el período de estabilización y 0,03 es la constante de solubilidad del $\mathrm{CO}_{2}$ en el plasma a $37^{\circ} \mathrm{C}$. Se considera normal el pHi menor que 7,35.

La interpretación correcta de los valores obtenidos exige eliminar toda fuente exógena de producción de $\mathrm{CO}_{2}$ en el estómago, como el proveniente de la titulación de la acidez gástrica, para lo que se utilizan bloqueadores $\mathrm{H}_{2}$, en nuestro caso ranitidina, $1 \mathrm{mg} \cdot \mathrm{kg} \cdot$ dosis ev, cada $8 \mathrm{~h}$, mientras dura la obtención de la muestrá.

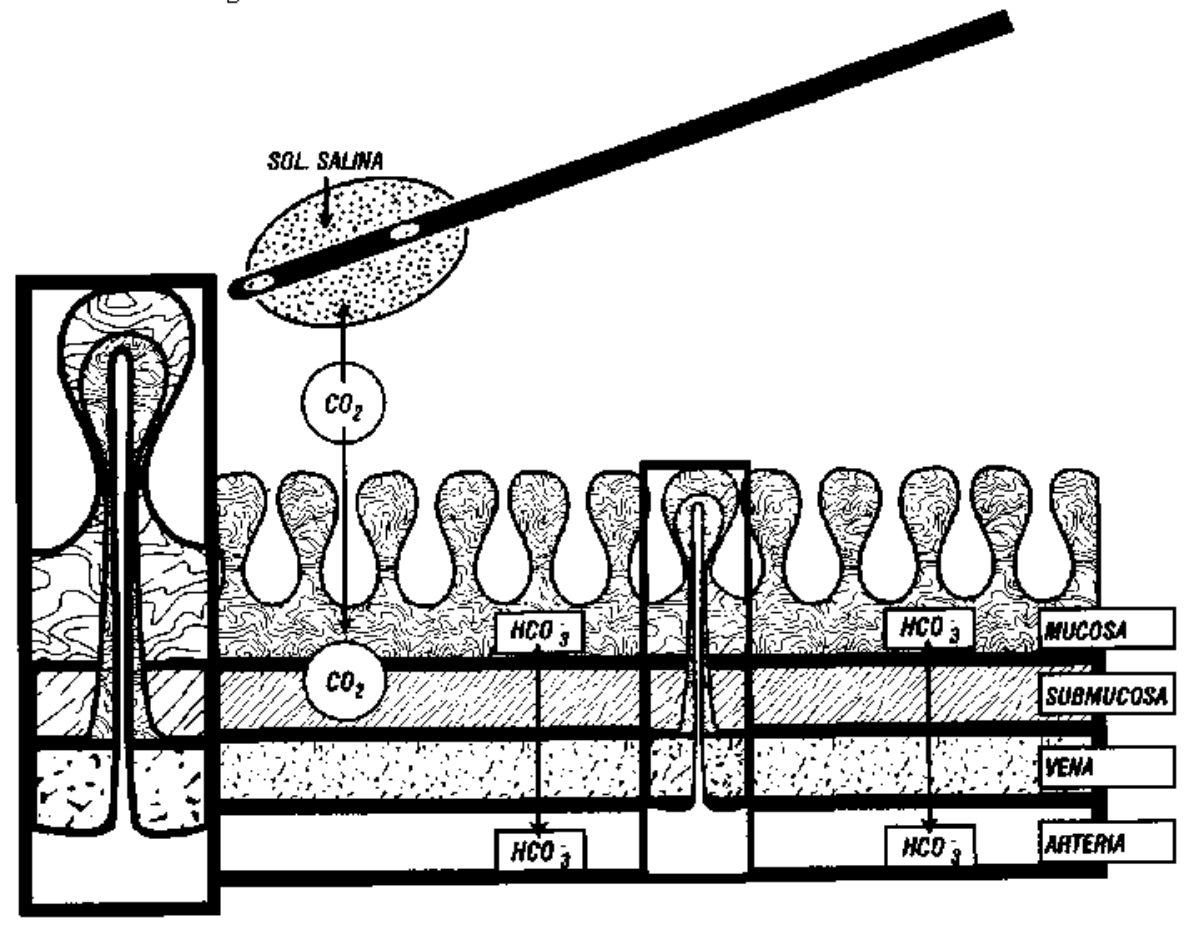

Figura 2: Representación esquemática de tonómetro a nivel de la mucosa gástrica. El $\mathrm{CO}_{2}$ de la nucusa difunde libremente hacia el balón de silicona Jográndose un equilibrio, cono el estabilecido entre las concenuraciones de bisarbonato en arteria y mucosa gástrica. 


\section{Indicaciones y contraindicaciones}

La mayor utilidad de la tonometría gástrica reside en la detección precoz de choque en la fase compensada, es decir, antes de la más mínima evidencia clínica de alteración hemodinámica sistémica.

En cstados descompensados de choque el mélodo aporta información adicional para el adecuado manejo del paciente. En la etapa de nornalización hemodinámica global, corroborada con $\mathrm{pHi}$ normal, permite suponer que, habiéndose normalizado lo que más tarda en recuperar, se ha asegurado una adecuada oxigenación tisular y que la resucitación cardiopulmonar ha sido efectiva.

Se recomienda emplear tonometría gástrica en pacientes que cursan con sepsis, especialmentc para el diagnóstico diferencial con síndrome séptico; en personas en riesgo de hipovolemia (por ejemplo, postoperatorio con peligro de sangramiento, traumalismo abdominal); en enfermos quirúrgicos que pudiesen sufrir isquemia mesentérica durante a después de la operación (p.e.: circulación extracorpórea); para titular el uso de ciertos fármacos vasoactivos, cuyo efecto vasoconstrictor pudiera ser deletéreo a tivel de la mucosa gastrointestinal, como la norepinefrina en pacientes con choque séptico: en la evaluación de la perfusión esplácnica en algunas condiciones clínicas relevantes (p.e.: faIla hepática fulminante). No se recomienda su utilización cuando hay riesgo de obstrucción mecánica del esófago, várices esofágicas, perforación esotágica o gástrica, hemorragia gastrointestinal recienle, jleo con reflujo biliar duodeno gástrico.

\section{Resultados}

En uuestra unidad comenzamos a emplear el método desde el año 1995. En el primer semestre se realizaron 291 mediciones en 39 pacientes (25 varoncs) de 6 meses a 15 años (promedio 5 años, DE 4 años 4 meses) de edad, que pesaban $21,6 \pm 17,9 \mathrm{~kg}$, márgenes 5 a $95 \mathrm{~kg}$. Se efectuaron entre 1 y 25 mediciones por paciente, promedio 7. El tonómetro estuvo instalado entre 1 y 9 días, promedio 2 . En 25 de los 39 pacientes el diagnóstico principal fue alguna infección grave como meningococcemia o neutropenia y síndrome séptico de diferentes etiologías. No se observaron complicaciones en relación a la técnica empleada en ninguno de los pacientes.

De los 25 pacientes con afecciones sépticas, en $10 \mathrm{el} \mathrm{pHi} \mathrm{era} \mathrm{anormal} \mathrm{al} \mathrm{ingreso,} \mathrm{normalizán-}$ dose en siete niños, que se recuperaron durante las $24 \mathrm{~h}$ siguientes. El pHi se mantuvo alterado en los otros tres enfermos, todos los cuales murieron.

Los principales patrones identificados al correlacionar el $\mathrm{pHi}$ con variables hemodinámicas y de laboratorio tradicionalmente empleadas fueron evoluciones clínica y de laboratorio compatibles con choque descompensado, que se correlacionaron $(r>0,85$ ) con exacerbación de la magnitud de la acidosis intramucosal; en otros caso la evaluación hemodinámica, de laboratorio y tonometria eran normales, como expresión de una circulación suficiente; la evaluación sistémica sugería en algunos pacientes una circulación estable, pero ellos tenían diferentes grados de acidosis intramucosal registrados por tonometría, reflejando un estado de choque compensado.

\section{Referencias}

1. Shomaket $W$ : Use and abuse of the baltoon tipe pulmonary artery (Swan-Ganz) catheter: Are patients get1ing their money's worth?. Crit Care Med 1992; 18: $1294-1296$

2. Gufiésrez G. Palizas F. Poglia G, el al: Gastric intramucosal $\mathrm{pH}$ as a therapeutic index of tissue oxygenation in critically ill patients. Lancet 1992: 339: 195-199.

3. Silepard $A P$. Kiel JW: A model of counter current shunting of oxygen in the intestinal villus. An J Physiol 1992; 262: HI 136.

4. Danztker D: The gastrointestinal tract: The canary of the body? JAMA 199.3; 270: 1247 .

5. Fidditn-Green RG: Stùdies in splanchnic ischemia and inultiple organ failure. In Marsion A. Bulkley GB, Fiddian-Green RG et al (Eds): Splanchnic ischemia and multiple organ failure. Si Louis, CV Mosby. 1989: 349363.

6. Fiddian.Gren RG, Amelin PM. Herrmann JB. et al: Prediction of the development of sigmoid ischemia in the day of aortic operations. Arch Surg 1986; 121:654.

7. Fiddian-Green RG. Pittenger $G$, Whitehouse WM: Back-diffusion of $\mathrm{CO} 2$ and its influence on the intramural pH in gastric mucosa. J Surg Res 1982; 33: 39. 
8. Antonsson $J B$, Boyle $C C$, Kryithoff $K L$, et as. Validation of tonometric measurement of gut intramural $\mathrm{pH}$ during endotoxemia and mesenteric occlusión jn pigs. Aln J Physiol 1990; 259: G 519.
9. Heand SO. Helsmourtel CM: Gastric tonometry in healthy volunteers: Effect of ranitidine on calculated intramural pH. Crit Core Med 1991; 19: 271-274.

\section{AVISO A LOS AUTORES}

Por acuerdo del Comité Editorial, Ia Revista Chílena de Pediatría devolverá sin tramitar todos los trabajos que no den estricto cumplimiento al Reglamento de Publicaciones y a las Instrucciones de los Autores que se editan en cada número de la Revista. 